\section{Can we predict and prevent postoperative morbidity in elderly patients?}

\section{Abhijit Talukdar, Jitin Yadav, Niju Pegu, Joydeep Purkayastha}

Dr. Bhubaneswar Borooah Cancer Institute, Guwahati, India

In their recent article [1], Wakahara et al demonstrated a significantly higher overall morbidity in elderly patients, with a higher incidence of anastomotic leak and cardiovascular complications. We want to highlight the role of risk assessment tools and calculators in predicting the outcome of surgery in elderly patients, as outcomes are more dependent on individual patients' parameters. We need to identify patients for whom surgery entails a greater risk, so that we can optimize their care and also pay more attention during the perioperative and postoperative periods. As clinicians, it is our duty to offer the maximum benefit to each and every patient.

The Physiological and Operative Severity Score for the Enumeration of Mortality and Morbidity (POSSUM) was designed for use in preoperative risk prediction, allowing for both individual physiological risk and the type of surgery performed [2]. Malnutrition has an estimated prevalence of $23 \%$ among the elderly and is associated with several contributing factors, such as altered metabolism, decreased appetite, medication use and chronic disease [3]. The Malnutrition Universal Screening Tool (MUST) combines both subjective and objective factors, such as body mass index, degree of weight loss and presence of acute disease, to determine the risk of malnutrition [4]. Enteral supplementation, including protein nutritional shakes, multivitamins and iron supplementation, involves simple interventions that may help support wound healing in the postoperative period [5].

The American College of Surgeons National Surgical Quality Improvement Program (ACS NSQIP) Surgical Risk Calculator accurately estimates the chance of a patient experiencing postoperative complications. In a study by Lubitz et al [6], it was found that ACS NSQIP accurately predicts outcomes for elective colorectal operations. We also intend to conduct a validation study to determine the role of ACS NSQIP in patients undergoing surgery for gastrointestinal cancers at our cancer center.

\section{References}

1. Wakahara T, Ueno N, Maeda T, et al. Postoperative morbidity in elderly patients after gastric cancer surgery. Ann Gastroenterol 2018;31:621-627.

2. Copeland GP, Jones D, Walters M. POSSUM: a scoring system for surgical audit. Br J Surg 1991;78:355-360.

3. Kaiser MJ, Bauer JM, Rämsch C, et al; Mini Nutritional Assessment International Group. Frequency of malnutrition in older adults: a multinational perspective using the mini nutritional assessment. J Am Geriatr Soc 2010;58:1734-1738.

4. Poulia KA, Yannakoulia M, Karageorgou D, et al. Evaluation of the efficacy of six nutritional screening tools to predict malnutrition in the elderly. Clin Nutr 2012;31:378-385.

5. Golladay GJ, Satpathy J, Jiranek WA. Patient optimization-strategies that work: malnutrition. J Arthroplasty 2016;31:1631-1634.

6. Lubitz AL, Chan E, Zarif D, et al. American College of Surgeons NSQIP risk calculator accuracy for emergent and elective colorectal operations. J Am Coll Surg 2017;225:601-611.

Department of Surgical Oncology, Dr. Bhubaneswar Borooah Cancer Institute, Guwahati, India

Correspondence to: Jitin Yadav, Department of Surgical Oncology, Dr. Bhubaneswar Borooah Cancer Institute, Guwahati, 781016 India, e-mail- jitindrcool@gmail.com

Conflict of Interest: None

Received 14 December 2018; accepted 27 December 2018; published online 18 January 2019

DOI: https://doi.org/10.20524/aog.2019.0353 